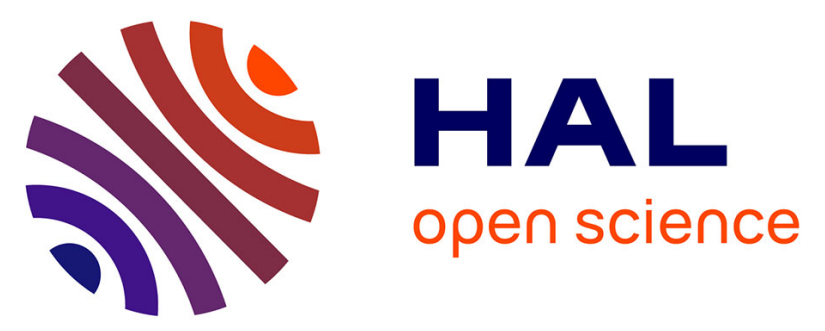

\title{
Superior Rate Capability and Cycling Stability in Partially Cation-Disordered Co-Free Li-Rich Layered Materials Enabled by an Initial Activation Process
}

Junghwa Lee, Yujeong Yang, Mihee Jeong, Nicolas Dupre, Maxim Avdeev, Won-Sub Yoon, Si-Young Choi, Byoungwoo Kang

\section{To cite this version:}

Junghwa Lee, Yujeong Yang, Mihee Jeong, Nicolas Dupre, Maxim Avdeev, et al.. Superior Rate Capability and Cycling Stability in Partially Cation-Disordered Co-Free Li-Rich Layered Materials Enabled by an Initial Activation Process. Chemistry of Materials, 2021, 33 (13), pp.5115-5126. 10.1021/acs.chemmater.1c01154. hal-03356025

\section{HAL Id: hal-03356025 \\ https://hal.science/hal-03356025}

Submitted on 7 Oct 2021

HAL is a multi-disciplinary open access archive for the deposit and dissemination of scientific research documents, whether they are published or not. The documents may come from teaching and research institutions in France or abroad, or from public or private research centers.
L'archive ouverte pluridisciplinaire HAL, est destinée au dépôt et à la diffusion de documents scientifiques de niveau recherche, publiés ou non, émanant des établissements d'enseignement et de recherche français ou étrangers, des laboratoires publics ou privés. 


\title{
Superior Rate Capability and Cycling Stability in Partially Cation-Disordered Co-Free Li-Rich Layered Materials Enabled by an Initial Activation Process
}

Junghwa Lee, Yujeong Yang, Mihee Jeong, Nicolas Dupre, Maxim Avdeev, Won-Sub

Yoon, Si-Young Choi, and Byoungwoo Kang

\begin{abstract}
Li-rich layered materials that have Co-free and Mnrich 3d-transition metals have the potential to increase the achievable energy density of batteries because they are inexpensive and yield high capacity by exploiting an additional oxygen redox reaction. However, these have low electrochemical activity and sustainability, with severe voltage fade, rapid capacity decay, and poor rate capability. Here, we report sustainable cycling stability and fast rate capability of Co-free $\mathrm{Li}_{2} \mathrm{MnO}_{3}$-based $\mathrm{Li}$-rich layered materials that are governed by the electrochemical activation process during the 1st cycle and that this process can be controlled by the degree of the cation disordering in the pristine material. From the comparative study of two samples that have different degrees of cation disordering in the same composition, an increase in cation disordering in the pristine material strongly improves its tolerance to structural changes in the bulk and on the surface during the activation process at the 1st cycle, leading to less structural changes for subsequent cycles. As a result, high electrochemical activity and superior rate capability in subsequent cycles can be achieved even with the cation disordering in the pristine. Furthermore, we verified the findings by developing an additional material that had higher cation disordering in the pristine structure than the samples tested and showing that the additional sample has improved rate capability and cycle retention. This understanding that sustainable electrochemical characteristics are governed by an activation process in the 1 st cycle, which can be controlled by a structural feature of the pristine material, will be useful in the design of low-cost, Li-rich layered materials that can achieve sustainable high energy density and fast rate capability for Li-ion batteries.
\end{abstract}

\section{INTRODUCTION}

Electric vehicles and electrical energy storage systems require inexpensive lithium-ion batteries (LIBs) that have high energy density.1,2 Li-rich layered materials $\left(\mathrm{Li}_{1+x} \mathrm{TM}_{1-x} \mathrm{O}_{2}, \mathrm{x}>0\right)$ that use Co-free 3d-transition metals (TMs) have been attracting a lot of attention because they can achieve much higher capacity at a lower cost than conventional TM redox-only materials that include Co, using both the TM redox reaction and oxygen redox reaction.3-6 However, Co-free 3d-TM-based Li-rich layered materials have several issues, including sluggish kinetics and poor electrochemical activity and sustainability with severe voltage fade and capacity fading during cycling. These problems are related to oxygen redox activity and sustainability. 7-9 The poor reversibility of the oxygen redox reaction is strongly coupled to TM migration in the Li-rich layered materials during electrochemical cycles. The coupled TM migration can cause irreversible structural changes during cycling 9,10 and thereby cause severe voltage decay and capacity fade upon cycling; these responses decrease the achievable energy density of the materials and hinder their practical applications.11 Approaches to solving these problems include doping with other elements such as cobalt,12 applying surface coatings,13,14 replacing 3d-TMs with 4d or 5d-TMs,5,15-18 or using cation-disordered rocksalt-type structures;19-21 the changes in the material properties of the pristine material such as the degree of cation disordering can improve the oxygen redox reaction activity and mitigate the structural changes during cycling.5,15-18,22,23 Co doping stabilizes the well-ordered layered structure in typical Co-free 3d-TM-based Li-rich layered materials and thereby improves the oxygen redox reaction and rate capability,24-26 but $\mathrm{Co}$ is expensive and toxic; therefore, it is not suitable for use in large-scale energy storage systems. Furthermore, poor electrochemical sustainability upon cycling is still a problem and is not well understood in Co-free 3d-TM-based Li-rich layered materials.

3d-TM-based Li-rich layered materials show a quite different voltage curve at the 1st cycle than in the subsequent cycles; therefore, electrochemical sustainability upon cycling can be affected by not only the properties of the pristine materials but also the electrochemical activation process that occurs during the 1st cycle. The 1 st cycle of a 3d-TM-based Li-rich layered material shows a voltage plateau at $\sim 4.5 \mathrm{~V}$ with a sloping voltage at the beginning of the charge and a sloping discharge voltage curve, whereas in subsequent cycles, the discharge/ charge curve has an "S"-shape without the voltage plateau at $\sim 4.5$ V.11,17,18 The difference indicates that the electrochemical reaction is quite different during the 1st cycle from that during subsequent cycles. During the 1st charge process, 3d-TM-based Li-rich layered materials undergo activation of the oxygen redox reaction, which yields the voltage plateau at $\sim 4.5 \mathrm{~V}$, and is accompanied by structural rearrangements or changes,11,27,28 whereas during subsequent cycles, this activation of the oxygen redox reaction is weak. As a 
result, the manner in which the oxygen redox reaction is activated and the way in which local structures change during the 1st cycle in 3d-TM-based Li-rich layered materials strongly affect the electrochemical activity and sustainability in subsequent cycles. For example, the irreversible oxygen redox reaction at the end of 1 st charge in the activation process can lead to the loss of different amounts of oxygen gas $27,29-32$ to the surface, and this difference can affect the electrochemical activity and sustainability in subsequent cycles. Oxygen loss can easily cause rearrangement of TMs in the surface and result in the formation of a densified surface layer $27,29-32$ that can block the reintercalation of $\mathrm{Li}$ in the following discharge process and subsequent cycles. The structural changes that occurred during the 1st cycle can trigger the structural changes in subsequent cycles. Severe structural changes in the activation process can drive the development of irreversible structural changes in subsequent cycles and cause low electrochemical sustainability. To improve sustainable electrochemical characteristics in 3d- TM-based Li-rich layered materials, the electrochemical activation process at the 1st cycle and its effects on the sustainable electrochemical characteristics in subsequent cycles should be well understood. Herein, we report that sustainable electrochemical characteristics of a Co-free 3d-TM-based Li-rich layered material are governed by the electrochemical activation during the 1st cycle and that this activation strongly depends on the structural feature of the pristine material. As a result, the different electrochemical activation during the 1 st cycle can affect not only the electrochemical activity at the 1st cycle but also the electrochemical reversibility and kinetics in subsequent cycles. One of such structural features is the amount of cation disordering ( $\mathrm{Ni}$ in Li layer) in the layered structure of the pristine material. The cation disordering of the pristine material remarkably improves the tolerance to structural changes in the bulk and on the surface during the activation process that occurred in the 1st cycle. In 3d-TM-based Li-rich layered materials with high cation disordering in the pristine material, the bulk during the 1st cycle is stabilized by suppressing the cation migration and negligible changes in Li local environments, and the surface is improved by forming a thin surface-reconstruction layer. We then applied these findings to fabricate an additional material by further increasing cation disordering in the pristine. The resulting material had the highest cation disordering in the pristine material and improved structural stability after the $1^{\text {st }}$ cycle; therefore, the material achieves remarkably improved sustainable electrochemical characteristics with fast kinetics during cycling.

\section{EXPERIMENTAL SECTION}

We aimed to determine how structural characteristics of pristine materials affect the activation process of the 1st cycle. We achieved this goal by comparing structural changes of the various samples that have the same composition but different structural features in the pristine.

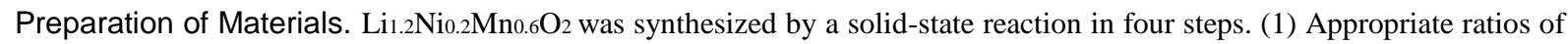
$\mathrm{Li}_{2} \mathrm{CO}_{3}, \mathrm{MnO}_{2}$, and $\mathrm{NiCO}_{3}$ were ball-milled in acetone for $12 \mathrm{~h}$. These samples had the molar ratio $\mathrm{Li} / \mathrm{Ni} / \mathrm{Mn}=1.2: 0.2: 0.6$. (2) A mix of precursors was pelletized and then calcined at $900{ }^{\circ} \mathrm{C}$ for $6 \mathrm{~h}$ in air. (3) The calcined pellets were ground and pulverized by planetary ball milling (PBM, Fritsch Pulverisette planetary ball mill) for $3 \mathrm{~h}$ to increase the reaction between the particles. The pulverized powder was repelletized. (4) The pellets were reannealed for $5 \mathrm{~h}$ in air at $800{ }^{\circ} \mathrm{C}$, then allowed to cool naturally to room temperature (RT) (sample name: relatively low cation disordered, LCD), or quenched to RT (sample name: cation disordered, CD) or reannealed for $5 \mathrm{~h}$ in air at $900{ }^{\circ} \mathrm{C}$, and then quenched to RT (sample name: relatively high cation disordered, $\mathrm{HCD}$ ).

Material Characterization. Neutron powder diffraction (NPD) measurements were performed on the Echidna highresolution powder diffractometer at the Australian Center for Neutron Scattering. The wavelength was $1.6215 \AA$ and the scan range was $5-165^{\circ}$ in increments of $0.05^{\circ}$. All data were collected at RT. The synchrotron X-ray diffraction measurements were performed on beamline 9B-HRPD at Pohang Accelerator Laboratory (PAL), Pohang, Korea. The incident X-rays were vertically collimated by a mirror and then monochromated to a wavelength of $1.4970 \AA$ by a double-crystal $\mathrm{Si}(111)$ monochromator. The datasets were collected in the range of $10^{\circ} \leq 2 \theta \leq 130^{\circ}$ in increments of $0.02^{\circ}$ ( $2 \theta$ range). Rietveld refinements were performed using FullProf software.

Nuclear Magnetic Resonance (NMR) Measurements. 6 Li NMR measurements were performed at RT on a Bruker Avance-200 spectrometer $\left(B_{0}=4.7 \mathrm{~T}\right.$, Larmor frequency $\mu_{0}=77.78 \mathrm{MHz}$ in $6 \mathrm{Li}$ resonance). Magic angle spinning (MAS) spectra were obtained using a Bruker MAS probe with a cylindrical 2.5-mm (od) zirconia rotor. Spinning speed was varied between 25 and $30 \mathrm{kHz}$ to determine the position of the isotropic resonances. 6Li MAS NMR spectra were acquired using an echo $(\pi / 2-\tau-\pi-\tau)$ pulse sequence with a $\pi / 2$ pulse of $2.3 \mu \mathrm{s}$. The recycle time was typically $0.5 \mathrm{~s}$. The isotropic shifts are relative to an external liquid $1 \mathrm{M}$ solution of $\mathrm{LiCl}$ set at $0 \mathrm{ppm}$.

X-ray Absorption Spectroscopy (XAS) Measurements. Hard X-ray absorption spectra of Mn and Ni K-edges were collected at the facility installed at beamline 7D at Pohang Light Source (PLS)-II in the transmission mode with the $\mathrm{N}_{2}$ gas- 
higher-order harmonics. The storage ring was operated at $2.5 \mathrm{GeV}$ with an injection current of $\sim 350 \mathrm{~mA}$. The spectral energies were calibrated using the first inflection points in the $\mathrm{Mn}$ and $\mathrm{Ni}$ metal foil spectra as references (i.e., Mn K-edge = $6539 \mathrm{eV}$ and Ni K-edge $=8333 \mathrm{eV}$ ). Data preprocessing operations such as deglitching, energy calibration, normalization, and leastsquares fitting with theory were performed using IFEFFIT, which used the FEFF code. Soft-XAS spectra of the O Kedge were recorded at beamline 8A1 - SPEM and 10D - XAS KIST of PLS-II in a total electron yield (TEY) mode and a fluorescence yield (FY) mode under high vacuum with a base pressure of $3 \times 10-10$ Torr. The spectral energy resolution was $0.1 \mathrm{eV}$, and the monochromator absorption features were normalized by dividing the detected signals by the photoemission current of a gold mesh placed in the incident beam. All absorption spectroscopy measurements were conducted at RT. Data preprocessing operations such as deglitching, energy calibration, normalization, and least-squares fitting with theory were performed using the WINXAS program with two or three Gaussian functions and one Arctangent function.

Scanning Transmission Electron Microscopy (STEM) Measurements. LCD and CD samples were observed in both pristine and 1st-charged states. Two samples of each state of each sample were prepared using the target surfacing system (TXP) and precision ion polishing (PIP). The mixed epoxy and powders were cured between typical Si substrates, mechanically ground to a thickness of $10 \mu \mathrm{m}$, and then ion milled using $\mathrm{Ar}_{+} . \mathrm{Li}$ extraction in the electrode material during charging induces the structural changes that occur at the surface and in bulk, which can be revealed by high angle annular dark-field (HAADF) and annular bright-field (ABF) STEM imaging. To visualize the local structure of the M phase in samples, we used a STEM (JEM-ARM200F, JEOL, Japan) at $200 \mathrm{kV}$ equipped with a fifth-order probe corrector (ASCOR,

CEOS GmbH, Germany). The optimum size of the electron probe for STEM observation was $\sim 0.8 \AA$. The collection semiangles of the HAADF detector were adjusted from 68 to $280 \mathrm{mrad}$ to collect scattered electrons at a large angle and obtain clear Zsensitive images. The images were obtained using a $6 \mathrm{~mm}$ aperture and a collection angle from 10 to $20 \mathrm{mrad}$. To reduce background noise (Filters Pro, HREM research Inc., Japan), the obtained raw images were processed using a bandpass Wiener filter with a local window.

Electrochemical Measurements. For electrochemical tests, composite electrodes (LCD, CD, or HCD) were made by mixing active material (80 wt \%), super-P carbon (Timcal, $15 \mathrm{wt} \%$ ), and binder (poly(vinylidene fluoride) (PVDF), $5 \mathrm{wt} \%$ ). The electrode of the HCD sample for the rate capability measurement was made by mixing the active material (70 wt \%), super-P carbon (Timcal, $25 \mathrm{wt} \%$ ), and binder (PVDF, $5 \mathrm{wt} \%$ ). A slurry mixture was tape-casted on Al foil using the doctor blade method. The loading density of the electrode was $2-4 \mathrm{mg} / \mathrm{cm} 2$. The cells were assembled in an Ar-filled glove box, then tested on a Maccor 2200 operating in a galvanostatic mode, with Li metal as an anode, nonaqueous electrolyte (1 M LiPF6) in ethylene carbonate (EC)/diethyl carbonate (1:1 by volume, PANAX ETEC Co. Ltd., battery grade), and Celgard 2400 as a separator in a 2032-coin cell. All cells were tested at RT.

\section{RESULTS AND DISCUSSION}

Structural Changes in Both Surface and Bulk of the Co-Free Li-Rich Layered Samples during the Activation Process in the 1st Cycle. Samples of $\mathrm{Co}$-free $\mathrm{Li}_{1.2 \mathrm{Ni}} \mathrm{Ni} 2 \mathrm{Mn} 0.6 \mathrm{O}_{2}$ were synthesized by a solid-state reaction with a usual natural cooling process after a high-temperature calcination process. It was labeled as an LCD (relatively low cation disordered) sample (detailed in Experimental Section). The Rietveld refinement result of neutron powder diffraction (NPD) (Figure 1a) data showed that the LCD sample was composed of twolayered phases; one is indexed to a rhombohedral phase ( $\mathrm{R}-3 \mathrm{~m}$ symmetry; $\mathrm{R}$ phase) such as $\mathrm{LiNi} .5 \mathrm{Mn} 0.5 \mathrm{O}_{2}$ (LNMO), and the other is indexed to a monoclinic phase ( $\mathrm{C} 2 / \mathrm{m}$ symmetry; $\mathrm{M}$ phase) such as $\mathrm{Li}_{2} \mathrm{MnO}_{3}$. The refinement result of NPD (Table S1a) indicates that the amount of Ni in the Li layer of the R phase in the sample is $\sim 0.057 \mathrm{~mol}$, which is a typical feature of the layered $\mathrm{R}$ phase prepared using a solid-state reaction. $33 \mathrm{In}$ contrast, the amount of $\mathrm{Ni}$ in the Li layer in the $\mathrm{M}$ phase in the sample was negligible. Scanning electron microscopy (SEM) image showed that the particles are irregularly faceted with primary particle size $>200 \mathrm{~nm}$ with agglomeration (Figure 1b). To understand the structural change of the samples during the 1st cycle, electrochemical tests (Figure S2a) were performed in the galvanostatic mode at a $\mathrm{C} / 20$ rate $(14 \mathrm{~mA} / \mathrm{g}$ ). During the 1 st charge, the LCD sample achieved $\sim 310 \mathrm{~mA} \cdot \mathrm{h} / \mathrm{g}$, leaving $\sim 0.25 \mathrm{~mol} \mathrm{Li}$ in the structure at the end of the charge with a long voltage plateau at $\sim 4.5 \mathrm{~V}$, which is related to an oxygen redox reaction. 6 After the 1st charge, the LCD sample achieved $\sim 235 \mathrm{~mA} \cdot \mathrm{h} / \mathrm{g}$ of discharge capacity and average discharge voltage of $3.45 \mathrm{~V}$ (i.e., the voltage at halfdischarge capacity or half-energy density during discharge) at 1 st discharge with Columbic efficiency of $\sim 75 \%$, which is consistent with previous reports.6,30 After the 1st cycle, the voltage profile at charge/discharge showed no voltage plateau. This irreversible change in the voltage profile after the 1st cycle is related to the activation of the oxygen redox reaction during the 1 st cycle. 


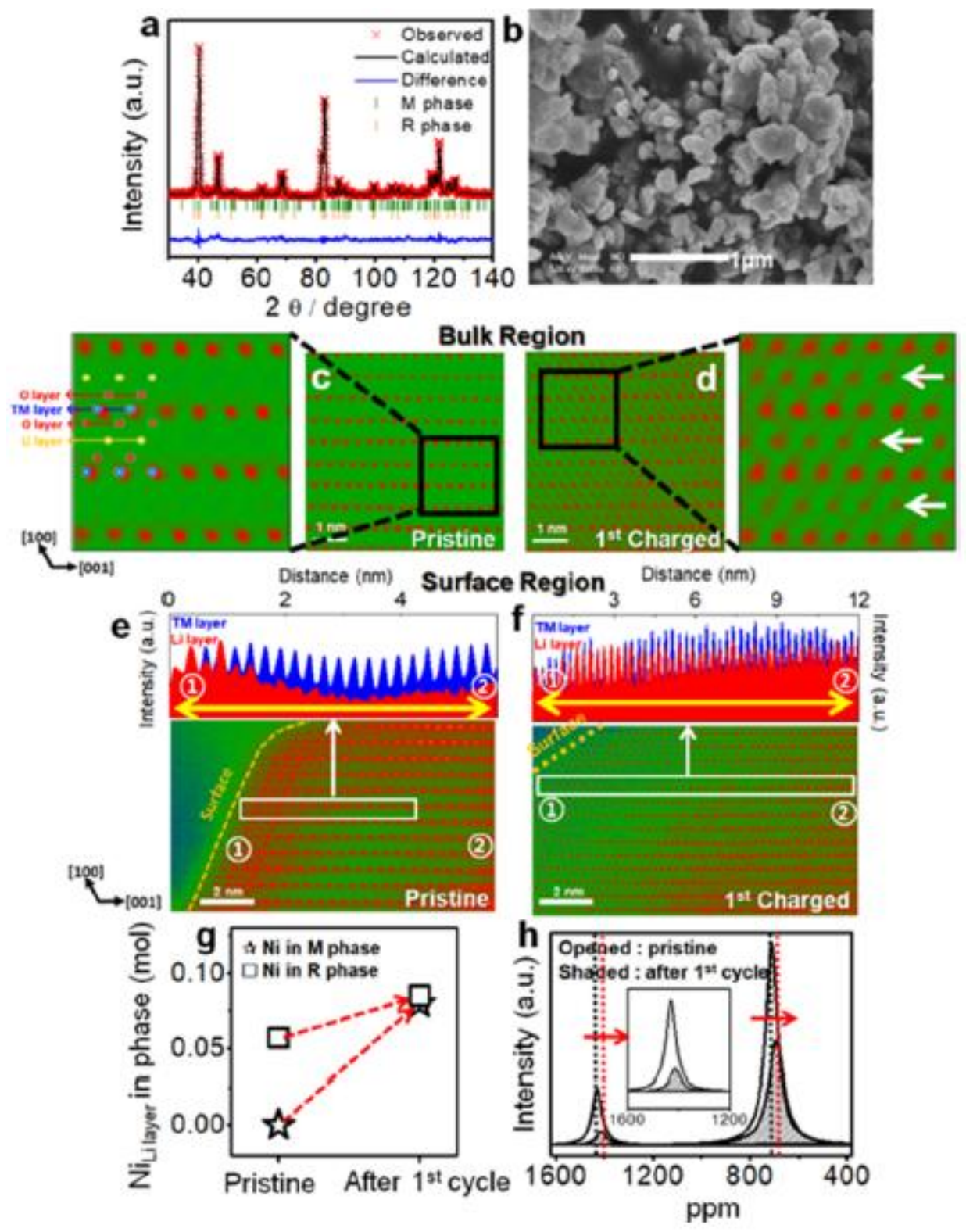

Figure 1. Structural characterization and structural changes during the 1st cycle in the LCD sample. (a) Rietveld refinement of the neutron powder diffraction (NPD) result of the sample. Vertical whiskers: theoretical NPD patterns of (orange) the R phase (e.g., LiNio.5 Mn0.5 2 ) and (green) the $\mathrm{M}$ phase (e.g., Li2 $\mathrm{MnO}_{3}$ ) (Table $\mathrm{S} 1 \mathrm{a}$ ). (b) SEM image of the sample. The HAADF-STEM image in the bulk region of the M phase is projected along the [010] direction in the (c) pristine state and the (d) 1st-charged state (white arrows: TM in the Li layer). The HAADF-STEM image in the surface region of the M phase along the [010] direction in the (e) pristine state and the (f) 1st-charged state. (g) Site occupancies of the Ni atoms in Li layers of both phases in the sample after the 1st cycle, as determined by the Rietveld refinement of NPD on ex situ electrodes (Figure S5a, Table S3a). (h) Change in Li local environments in the M phase in the sample after the 1st cycle at C/20-C/20 by 6Li MAS NMR measurements of ex situ electrodes (Figure S5d,e).

To understand the contribution of redox reactions in the LCD sample, X-ray absorption spectroscopy (XAS) measurements (Figure S2b-d) and soft X-ray absorption spectroscopy (SXAS) measurements (Figure S3, Table S2a) $4,6,34$ were obtained during the two cycles. The LCD sample had $\mathrm{Ni}_{2+}$ and $\mathrm{Mn}_{4+}$ in the pristine structure that is similar to the reported results of $\mathrm{Ni}_{2+}(\sim 8350 \mathrm{eV})$ and $\mathrm{Mn}_{+}(\sim 6560 \mathrm{eV}) .4,6,34$ During the 1st charge, $\mathrm{Ni}_{2+}$ is oxidized to $\mathrm{Ni}_{4+}(8355 \mathrm{eV})$, whereas $\mathrm{Mn}_{4+}$ changes little, then during the $1^{\text {st }}$ discharge, $\mathrm{Ni}_{4+}$ is reduced back to 
$\mathrm{Ni}_{2+}$, but $\mathrm{Mn}_{4+}$ does not change. These observations indicate that the charge capacity in the LCD sample is caused only by the $\mathrm{Ni}$ redox reaction from $2+$ to $4+$, without any redox reaction by $\mathrm{Mn}$; this result is consistent with reported results.4,34 However, the increase in the cation disordering only in the R phase in the LCD sample may cause less TM redox reaction activity than a theoretical capacity of $\sim 125 \mathrm{~mA} \cdot \mathrm{h} / \mathrm{g}$ of Ni redox.4,34 The LCD sample shows that the redox reaction of Ni occurs mainly in the LNMO phase and that some of the $\mathrm{Ni}$ in the $\mathrm{Li}$ layer, which is the amount of the cation disordering, can affect the kinetic properties and result in not well activated Ni redox. As a result, the oxygen redox reaction in the LCD sample can contribute an additional discharge capacity of $\sim 110 \mathrm{~mA} \cdot \mathrm{h} / \mathrm{g}$ to the obtained discharge capacity during the two cycles (Figure S2a).

Further evidence for the oxygen redox reaction is given by the observation of a pre-edge region below $\sim 534 \mathrm{eV}$ in the O K-edge SXAS spectra in the total electron yield (TEY) mode (Figure S3, Table S2a). This region can be attributed to the transition from $\mathrm{O} 1 \mathrm{~s}$ to the unoccupied state in the $\mathrm{O} 2 \mathrm{p}$ orbital and from $\mathrm{O} 1 \mathrm{~s}$ to the empty hybridized TM dO 2p orbitals.4,35 The integrated intensity of the O K-edge at $<534 \mathrm{eV}$ during the two cycles in the LCD sample is higher in the charged state than in the pristine state and in the discharged state (Figure S3). This phenomenon indicates that the oxygen redox reaction in the LCD sample is active and reversible. However, in the 2nd cycle, the contribution of the oxygen redox reaction is much lower than that in the 1st cycle; this change indicates a decrease in the oxygen redox activity. The oxygen redox reaction in 3d-TM-based Li-rich layered materials occurs mainly in the $\mathrm{M}$ phase, which has excess $\mathrm{Li}$; $36-38$ therefore, the low oxygen redox activity during the 2 nd cycle in the LCD sample may be a result of the irreversible structural change during the 1 st cycle. To determine how the activation process during the $1^{\text {st }}$ cycle affects the electrochemical properties of the samples (Figure 1c-f), the changes in local structures in the bulk and at the surface after the 1st charge were measured using STEM. The observations are focused on the structural changes of the $M$ phase before and after the 1st charge because this phase in 3d-TM-based Li-rich layered materials generally undergoes severe structural changes caused by the oxygen redox reaction in the 1st cycle or during cycling.10,30,36,39 The HAADF-STEM image visualizes only heavy atoms such as $\mathrm{Ni}$ and $\mathrm{Mn} ; 10,30,36,39$ hence, HAADF-STEM images (Figure 1c) show that the LCD sample does not have any TM in the Li layer of the M phase in the pristine structure and, therefore, has negligible cation disordering in the M phase. This result is consistent with the NPD refinement result (Table S1a). However, after the 1st charge, HAADF-STEM images of the LCD sample (Figure 1d) showed continuous bright contrast in the Li layer; this result indicates that a substantial amount of TM ions migrated into Li layers during the 1st charge. The M phase in the LCD sample undergoes severe local structural changes in the bulk during the 1st charge. The ABFSTEM mode can visualize light elements such as $\mathrm{O}$ and Li; observations of the LCD sample (Figure S4) show that after the 1st charge, the Li layers showed continuous bright contrast of TMs, although these layers should be empty at the end of the charge due to a large amount of Li extraction. This observation indicates that many TM ions migrated into the Li layer. This migration may be caused by the intrinsic instability of the layered structure or by the activation of the oxygen redox reaction. These possibilities indicate that the cation disordering, i.e., TM in the Li layer, in the $\mathrm{M}$ phase, is electrochemically created and increases because of the structural changes that occur during the initial activation process in the 1st charge. This suggestion is consistent with the typical feature that is observed in 3d-TM-based Li-rich layered materials.10,30 The lowering of oxygen redox activity in the $2^{\text {nd }}$ cycle can be ascribed to the severe structural change in the $1 \mathrm{st}$ charge.

More importantly, in 3d-TM-based Li-rich layered materials, the structural change in the surface region is more severe than in the bulk region because the reconstruction reactions such as TM ion migration to the surface and surface densification induced by the oxygen gas release occur during the $1^{\text {st }}$ charging process.29,30,32 We also observed severe structural changes on the surface region after the 1st charge in the LCD sample (Figure 1e,f). HAADF-STEM images and their intensity profiles on the surface in the pristine sample changed after the $1^{\text {st }}$ charge. Even if the LCD sample does not achieve full extraction of Li (Figure S2a), it forms a thick surface reconstruction layer with a large amount of migration of TM ions up to $12 \mathrm{~nm}$ into the Li layer (Figure 1f, yellow arrow) from the pristine surface (Figure 1e, yellow arrow); this observation is consistent with previous results.29,30,32 Continuous loss of the oxygen gas, concurrent with a large amount of Li extraction and the consequent migration of TM ions during the 1st charge, could result in thick surface densification. These STEM results clearly illustrate that during the 1st charge, the $\mathrm{M}$ phase in 3d-TM-based Li-rich layered material undergoes severe structural changes, both in the bulk structure region and near the surface.

To understand the structural changes in bulk and Li local environments after the activation process in the $1^{\text {st }}$ cycle, NPD (Figure 1g) was measured on ex situ electrodes of the LCD sample after the 1st cycle. Rietveld refinement results (Figure S5a, Table S3a) of NPD on these electrodes show that the LCD sample after the $1^{\text {st }}$ cycle had significantly increased cation disordering ( $\mathrm{Ni}$ in Li layer) in the $\mathrm{M}$ phase (Figure $1 \mathrm{~g}$, star) compared to the R phase (Figure 1g, square). This result occurred because of severe TM migration into the Li layer during the 1 st cycle, as suggested by the STEM results (Figure 1e). During the activation process that occurred in the $1^{\text {st }}$ cycle, the LCD sample underwent significant structural changes because of the increase in electrochemically 
created cation disordering and severe migration of the TM ions into the Li layers in both bulk (Figure 1d) and surface regions (Figure 1f).

Further evidence of structural changes in the LCD sample after the 1 st cycle is provided by Li local environments obtained using $6 \mathrm{Li}$ NMR measurements on ex situ electrodes (Figures $1 \mathrm{~h}$ and $\mathrm{S} 5 \mathrm{~d}, \mathrm{e}$ ). Li in the sample encountered four main local environments: $\mathrm{Li}$ in Li layers at $\sim 530 \mathrm{ppm}$ and TM layers at $\sim 1290 \mathrm{ppm}$ in the $\mathrm{R}$ phase, and $\mathrm{Li}$ in $\mathrm{Li}$ layers at $\sim 700 \mathrm{ppm}$ and TM layers at $\sim 1420 \mathrm{ppm}$ in the M phase.34,35 During the activation process in the 1 st cycle, the LCD sample showed overall changes in the peak shifts of Li that correspond to the Li layer and the TM layer of both M and R phases (Figure S5e). These changes in local Li environments in the LCD sample can be a result of the migration of TM ions during the 1st cycle, especially during the 1st charge via the increased cation disordering. The shifts to the lower frequency of $\mathrm{Li}$ in the $\mathrm{Li}$ layer at $\sim 700 \mathrm{ppm}$ and the TM layer at $\sim 1420 \mathrm{ppm}$ may be a result of an overall change in the $\mathrm{Mn} / \mathrm{Ni}$ ratio near $\mathrm{Li}$ as a result of the TM migration.40,41 These observations show that the activation process in the 1st cycle in the LCD sample substantially changes the local Li environments and causes severe structural changes in the bulk region, in which a large amount of TM migration occurs (Figure 1d), and to densification of the surface region (Figure 1f) in the $M$ phase. After the 1st cycle, the 3d-TM-based Lirich layered materials can have quite different structural/ chemical features from the pristine due to the increased cation disordering and changed Li local environments caused by the activation process in the 1st cycle. After 20 cycles, 6 Li NMR measurements on the cycled LCD sample (Figure S5f) clearly show that the Li local environments change significantly in the M phase after the 1st cycle continuously occurs during the 20th cycle. As a result, these structural/chemical changes that occurred in the 1st cycle strongly affect electrochemical sustainability and activity and rate capability of the $3 \mathrm{~d}$ TMbased Li-rich layered materials in subsequent cycles. For example, severe structural changes during the activation process in the 1st cycle can lead to poor electrochemical sustainability and fading of energy density during cycling. 27,28,31,42 This finding suggests that sustainable electrochemical characteristics of the 3d-TM-based Li-rich layered materials after the 1 st cycle depend strongly on the structural changes that occur during the $1^{\text {st }}$ cycle. These structural changes should be controlled and improved for achieving high electrochemical performance.To understand how the structure of pristine 3d-TM-based Li-rich layered materials affects structural changes during the activation process in the 1st cycle, we prepared the CD sample, which had the same chemical composition as the LCD sample, but different degrees of the cation disordering in the M phase were obtained using a quenching process after a high temperature calcination process. The refinement results of NPD (Figure 2a, Table S1b) indicate that the CD sample is composed of two-layered R and M phases, like the LCD sample. However, the CD sample has quite different Ni distribution over the two phases, R and M phases; therefore, the amount of $\mathrm{Ni}$ in the Li layer increased (cation disordering), especially in the $\mathrm{M}$ phase. The amount of $\mathrm{Ni}$ in the $\mathrm{Li}$ layer of the $\mathrm{R}$ phase in both samples is similar to each other, i.e., $\sim 0.057 \mathrm{~mol}$ for the LCD sample and $\sim 0.060$ mol for the CD sample. In contrast, the amount of $\mathrm{Ni}$ in the Li layer in the $\mathrm{M}$ phase in both samples is quite different; the CD sample had $\sim 0.080 \mathrm{~mol} \mathrm{Ni}$ in the Li layer in the M phase, whereas the LCD sample had a negligible amount, i.e., the CD sample had a much higher cation disordering in the pristine sample than the LCD sample, even though the two samples have a similar particle size, morphology (Figure 2b), and overall composition (Table S4).

To study the effect of the increase in the cation disordering in the pristine sample in the 3d-TM-based Li-rich layered materials on the activation process in the 1st cycle, the voltage profiles of the CD sample (Figure S6a) were evaluated in the galvanostatic mode, as for the LCD sample (Figure S2a). During the 1st charge, the CD sample achieved a charge capacity of $\sim 390 \mathrm{~mA} \cdot \mathrm{h} / \mathrm{g}$, which is close to a theoretical capacity of $\sim 385 \mathrm{~mA} \cdot \mathrm{h} / \mathrm{g}$. Even if almost all of the $\mathrm{Li}$ is extracted from the pristine structure during the 1 st charge, the CD sample can achieve a discharge capacity of $\sim 300 \mathrm{~mA} \cdot \mathrm{h} / \mathrm{g}$, which is much higher than that of the LCD sample, and the average discharge potential of $3.58 \mathrm{~V}$, which is higher than that of the LCD sample, $\sim 3.45 \mathrm{~V}$. The two samples show similar 1 st-cycle Columbic efficiency, $\sim 75 \%$, for the LCD sample and $\sim 77 \%$ for the CD sample, but the absolute amount of extraction and insertion of $\mathrm{Li}$ is much higher in the CD sample than in the LCD sample; this difference indicates that the electrochemical activity of the CD sample is remarkably increased.

The CD sample retained high reversible discharge capacities of $\sim 300 \mathrm{~mA} \cdot \mathrm{h} / \mathrm{g}$ with a high discharge voltage of $3.58 \mathrm{~V}$ for two cycles (Figure S6a). Given that the two samples have similar particle size and morphology (Figures $1 \mathrm{~b}$ and 2b) and similar overall composition (Table S4), these results indicate that increased cation disordering in the $\mathrm{M}$ phase in the pristine $\mathrm{CD}$ sample can improve electrochemical activity during the $1^{\text {st }}$ cycle. 
Ex situ XAS (Figure S6b-d) and ex situ SXAS (Figure S7, Table S2b)4,6,34 of the CD sample were also carried out during the two cycles to understand the redox reactions. The oxidation states of $\mathrm{Ni2} / \mathrm{Ni4}+$ and $\mathrm{Mn}$ in the $\mathrm{CD}$ sample changed in the same as in the LCD sample during the electrochemical cycle; this similarity indicates that both samples obtain a similar contribution of $\sim 125 \mathrm{~mA} \cdot \mathrm{h} / \mathrm{g}$ from the Ni redox reaction from $2+$ to $4+$, to the obtained capacity without any redox reaction of $\mathrm{Mn}$; this result is consistent with the reported results.4,34,43 As a result, the oxygen redox reaction in the $\mathrm{CD}$ sample must be the source of the additional $\sim 175 \mathrm{~mA} \cdot \mathrm{h} / \mathrm{g}$ of reversible discharge capacity to the obtained discharge capacity; this inference suggests that the reversible oxygen redox activity is substantially improved and then much higher than in the LCD sample, in which the oxygen redox reaction contributed less than $\sim 110 \mathrm{~mA} \cdot \mathrm{h} / \mathrm{g}$ during the two cycles (Figures S2a and S6a). Further evidence of the increased oxygen redox activity and sustainability is provided by the SXAS data for two cycles (Figure S7), which shows that the oxygen redox reaction is much higher in the CD sample than in the LCD sample. Given that the $\mathrm{CD}$ sample has a much higher degree of the cation disordering in the $\mathrm{M}$ phase of the pristine than the LCD sample, the improved electrochemical activity and sustainability in the CD sample in the 1 st cycle indicate that the activation process is strongly affected by the structural features of the pristine such as the cation disordering.

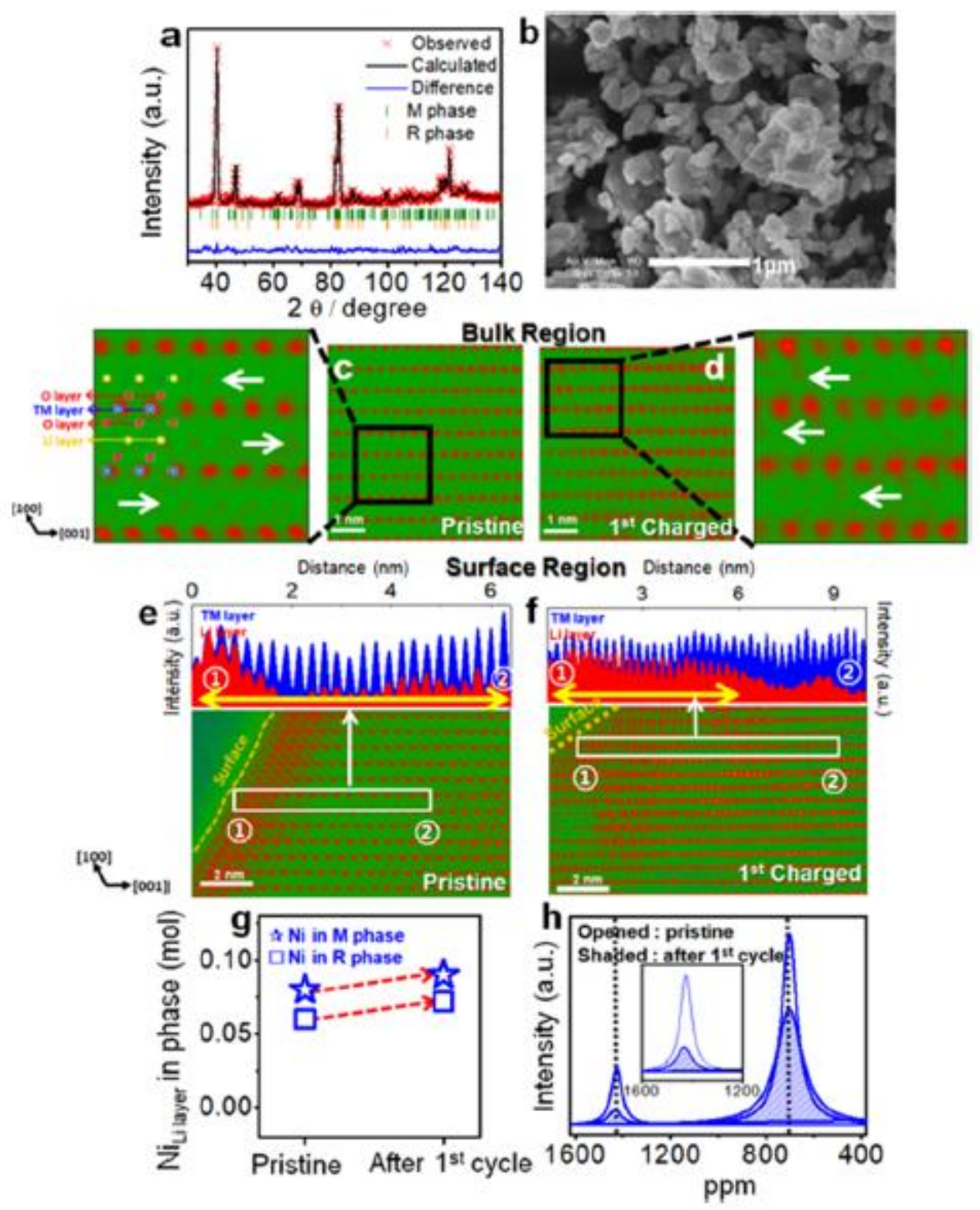

Figure 2. Structural characterization and structural changes during the 1st cycle in the CD sample. (a) Rietveld refinement of the neutron powder diffraction (NPD) result of the sample. Vertical whiskers: theoretical NPD patterns of (orange) the R phase (e.g., LiNio.5 $\mathrm{Mn}_{0.5} \mathrm{O}_{2}$ ) and (green) the $\mathrm{M}$ phase (e.g., $\mathrm{Li}_{2} \mathrm{MnO}_{3}$ ) (Table $\mathrm{S} 1$ b). (b) SEM image of the sample. The 
HAADF-STEM image in the bulk region of the M phase is projected along the [010] direction in the (c) pristine state and the (d) 1st-charged state (white arrows: TM in the Li layer). The HAADF-STEM image in the surface region of the M phase along the [010] direction in the (e) pristine state and the (f) 1st-charged state. (g) Site occupancies of Ni atoms in Li layers of both phases in the sample after the 1st cycle, as determined by the Rietveld refinement of NPD on ex situ electrodes (Figure $\mathrm{S} 10 \mathrm{a}$, Table S3b). (h) Change in Li local environments in the $\mathrm{M}$ phase in the sample after the 1 st cycle at $\mathrm{C} / 20-\mathrm{C} / 20 \mathrm{by} 6 \mathrm{Li}$ MAS NMR measurements of ex situ electrodes (Figure S10b,c).

To determine how the cation disordering in the pristine sample affects the activation process during the 1st cycle in which a large amount of $\mathrm{Li}$ is extracted, the changes in local structures in the $\mathrm{M}$ phase from the bulk to the surface after the 1st charge were measured using STEM (Figure 2c-f). HAADF-STEM images (Figure S8) of the pristine structure of the CD sample (Figure 2c) show distinct TM contrast in the Li layer of the M phase; this result is evidence of a certain amount of the cation disordering from the $\mathrm{M}$ phase of the pristine. Given that light elements such as $\mathrm{Li}$ and oxygen are weakly visualized using the HAADF-STEM mode, 10,24,30,33 the observed TM contrast in the Li layer in the pristine structure of the $\mathrm{CD}$ sample can be attributed to the presence of transition metals such as $\mathrm{Ni}$ and $\mathrm{Mn}$. Ni2+ions in the layered structure can easily move to the $\mathrm{Li}$ layer; 44 therefore, $\mathrm{Ni}$ atoms could enter the Li layers and thereby cause an increase in the cation disordering in the M phase; this possibility is consistent with the NPD refinement result (Table S1b). Surprisingly, after the 1st charge, the TM contrast in the Li layer barely changed in the M phase of the CD sample (Figure 2d) even though Li ions were almost fully extracted (Figure S6a) and a much higher oxygen redox reaction occurred in the 1st charge than in the LCD sample. The negligible changes in TM contrast between the pristine structure and the structure after the 1st charge in the $\mathrm{M}$ phase in the $\mathrm{CD}$ sample indicate that the cation disordering in the pristine state of the $\mathrm{M}$ phase in 3d-TM-based Li-rich layered materials can suppress the migration of TM ions during the 1st charge. The layered structural instability plays a critical role in driving the migration of TM ions when sufficient $\mathrm{Li}$ ions are extracted because of the presence of a large number of vacant Li sites.9,10 However, the cation disordering in the pristine sample decreases the amount of vacant $\mathrm{Li}$ sites even with full extraction of $\mathrm{Li}$ because of $\mathrm{Ni}$ in $\mathrm{Li}$ sites in the $\mathrm{Li}$ layer and thereby can substantially improve the stability of the layered structure and the tolerance to the structural changes that occur during the activation process; this tolerance leads to negligible structural changes in the local structure. After the 1st charge, the ABF-STEM image of the CD sample (Figure S9) showed almost no $\mathrm{Li}$ contrast in the $\mathrm{Li}$ layer, whereas TM contrast in the Li layer, because of cation disordering in the pristine sample, can be identified even after almost full extraction of Li. The STEM data clearly shows that the CD sample underwent negligible structural changes during the activation process in the 1st charge, unlike the LCD sample, which underwent severe structural changes. This difference in structural changes during the 1st charge indicates that the cation disordering in the pristine sample, especially of the $\mathrm{M}$ phase, will help reduce structural changes in the bulk and thereby significantly increase the electrochemical activity in the 1st charge, and lead to full extraction of Li.

The structural change in the surface region of the CD sample (Figure 2e,f) was substantially mitigated even after almost full $\mathrm{Li}$ extraction during the activation process in the $1^{\text {st }}$ charge, compared to the change in the LCD sample (Figure1e,f). HADDF-STEM images and their intensity profiles on the surface after the 1st charge show quite different surfacereconstruction behavior compared to the LCD sample. The CD sample does not show severe surface-reconstruction reaction while maintaining a thin $(\sim 6 \mathrm{~nm})$ surfacereconstruction layer (Figure $2 \mathrm{f}$, yellow arrow) from the pristine surface (Figure 2e, yellow arrow), which is much less than that at the surface of the LCD sample (Figure 1f, yellow arrow). Even though the amount of extracted Li is much higher in the CD sample than in the LCD sample during the 1st charge (Figures S2a and S6a), the structural change in the surface region of the CD sample is significantly reduced compared to the LCD sample. These STEM results illustrate that the increase in the cation disordering in the $\mathrm{M}$ phase in pristine 3d-TM-based Li-rich layered materials significantly mitigates the structural changes that occurred in the activation process during the 1st charge, both in the bulk structure and at the surface.

Refinement results of NPD on the ex situ CD electrodes after the 1st cycle, as shown in Figure 2g (Figure S10a, Table S3b), show that the cation disordering barely increased in both R (Figure $2 \mathrm{~g}$, square) and M phases (Figure $2 \mathrm{~g}$, star). This negligible increase in the cation disordering during the $1^{\text {st }}$ cycle is due to suppressed TM migration after the 1st charge, as observed in STEM results (Figure 2d). The CD sample has a remarkably improved tolerance to structural changes during the 1st cycle even though it utilizes much more Li than the LCD sample during the 1st cycle. These results show that the activation process during the 1 st cycle in the CD sample is substantially improved by the cation disordering in the pristine sample leading to the mitigated structural changes in both bulk and surface regions leading to high electrochemical activity in the 1st cycle. As a result, negligible structural changes and the improved electrochemical activity in the 1st cycle in the CD sample have positive effects on the electrochemical activity and achievable capacity in subsequent cycles.

6Li NMR measurements on ex situ electrodes (Figures $2 \mathrm{~h}$ and S10c,d) show that the CD sample has a negligible change in the Li local environments during the 1st cycle and after the 20th cycle unlikely the LCD 
sample, which has distinct changes in the Li peaks that correspond to the Li layer and the TM layer in both M and $\mathrm{R}$ phases (Figures $1 \mathrm{~h}$ and S5e,f). This indicates that the negligible changes in the Li local environments and suppressed TM migrations into the Li layer in the activation process in the 1st cycle can be maintained during cycles, partly because the cation disordering in the pristine sample can improve the tolerance to structural changes during electrochemical activity. As a result, electrochemical activity and electrochemical sustainability in the 1st and later cycles (Figure S6a) in the CD sample can be improved.

Electrochemical Characteristics of the Samples after the Activation Process in the 1st Cycle. The two samples underwent quite different structural changes during the activation process in the 1st cycle. The LCD sample (Figure 3a), which does not have any cation disordering in the pristine sample in the M phase, underwent severe structural changes in both the surface region and the bulk region because of TM migration into Li layers during the 1st charge and to change in the local environment of Li after the 1st cycle; these processes led to limited electrochemical activity and the increase in the electrochemically created cation disordering after the $1 \mathrm{st}$ cycle. In contrast, the CD sample in Figure 3b, which has the cation disordering in the pristine sample in the M phase, undergoes little structural changes in the bulk and the surface, with negligible TM migration into the Li layer during the 1st charge, and little changes in Li local environments during the 1st cycle. As a result, the LCD sample shows limited electrochemical activity and sustainability (Figure S2a), but the CD sample shows enhanced electrochemical activity (Figure S6a).

To understand the effects of the structural changes in the activation process during the 1 st cycle on the redox potential and achievable capacity of the 1 st cycle, the voltage curve (Figure 3c) and the $\mathrm{dQ} / \mathrm{dV}$ profile (Figure $3 \mathrm{~d})$ at the 1 st cycle were compared. The CD sample showed substantially improved electrochemical activity and much lower reaction potential of the oxygen redox reaction potential at the voltage plateau in the 1st charge than in the LCD sample. Consequently, the CD sample during the 1st discharge showed a much higher average discharge potential $=3.58 \mathrm{~V}$ and a much higher discharge capacity than the LCD sample (Figure 3c). This indicates that the structural changes that occur during the activation process in the 1st cycle strongly affect electrochemical activity, which can set the achievable capacity in the 1st cycle, and the discharge redox potential of the 3d-TM-based Li-rich layered materials.

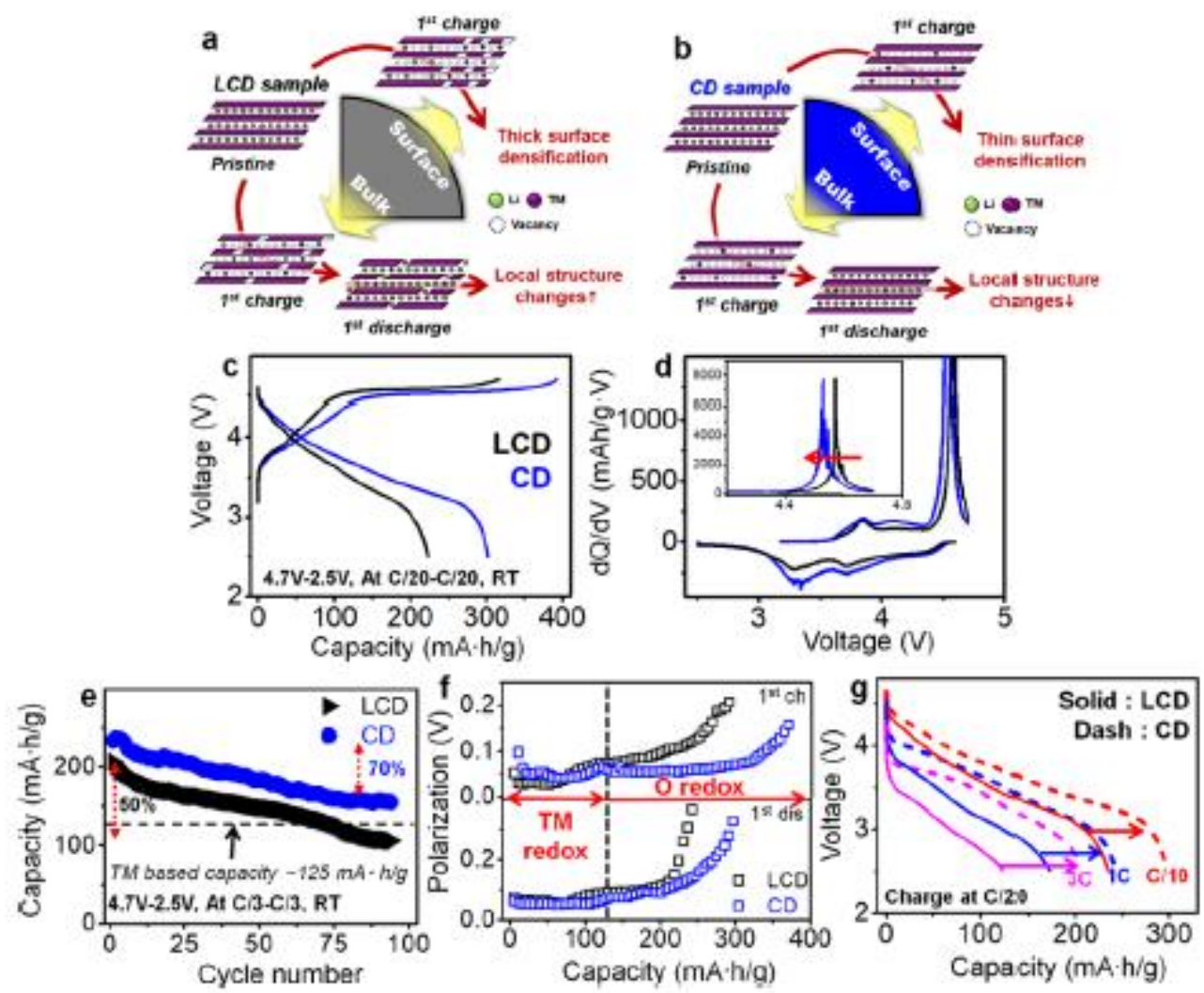

Figure 3. Electrochemical properties in the 1st cycle and in subsequent cycles and kinetic behaviors in the LCD and CD samples. Schematic diagram of structural changes from pristine to 1 st charge and after the 1st cycle, obtained by reference to HAADF-STEM images of the (a) LCD sample and the (b) CD sample. Compared $1^{\text {st }}$ electrochemical behavior of both samples, (c) voltage profile and (d) $\mathrm{dQ} / \mathrm{dV}$ profile at $2.5-4.7 \mathrm{~V}$ and $\mathrm{C} / 20(14 \mathrm{~mA} / \mathrm{g})$ at $\mathrm{RT}$. (e) Capacity retention of the 
samples at the $\mathrm{C} / 3$ charge and $\mathrm{C} / 3$ discharge rates for cycles after the activation process in the 1 st cycle at the $\mathrm{C} / 20$ charge and discharge rate. (f), Polarization during the 1 st cycle from galvanostatic intermittent titration technique (GITT) curves at 2.5- $4.7 \mathrm{~V}$ and $\mathrm{C} / 50(5.6 \mathrm{~mA} / \mathrm{g})$ at RT. (g) Rate capability of the samples at different discharge rates with the same charge rate at $\mathrm{C} / 20$. All electrochemical tests were performed at room temperature, with an operating voltage window from 2.5 to 4.7 V.

The structural features such as the degree of the cation disordering in the pristine sample in 3d-TM-based Li-rich layered materials can strongly improve the tolerance to the structural changes that occur during the 1st charge and thereby yield negligible structural changes in subsequent cycles. As a result, 3d-TM-based Li-rich layered materials that have an increased amount of cation disordering in the pristine sample can undergo a negligible structural change during the 1st cycle; therefore, the sample retains high discharge voltage, low oxygen redox potential plateau, and high achievable capacity in the 1st cycle. After the 1st cycle, the two samples had quite different degrees of structural features because they undergo different activation processes in the 1st cycle; the CD sample starts with negligible structural changes after the 1st cycle and has improved tolerance to the structural changes that occur in the subsequent cycles. However, the LCD sample starts with severe structural changes after the 1 st cycle and barely has the tolerance to the structural changes that occurred in subsequent cycles. These different starting points with respect to the structural changes and the tolerance in the two samples significantly affect the electrochemical activity and sustainability in subsequent cycles.

The CD sample shows much better capacity retention in subsequent cycles (Figure 3e) and less voltage fade than the LCD sample. The LCD sample at C/3 charge and C/3 discharge rate showed a rapid capacity fade from $\sim 200$

to $\sim 100 \mathrm{~mA} \cdot \mathrm{h} / \mathrm{g}(\sim 50 \%)$ for 90 cycles. In contrast, the CD sample showed improved capacity retention from 230 to $\sim 160 \mathrm{~mA} \cdot \mathrm{h} / \mathrm{g}(\sim 70 \%)$ during 90 cycles, which is higher capacity than the TM-based redox capacity ( 125 $\mathrm{mA} \cdot \mathrm{h} / \mathrm{g}$ ), and achieved a low voltage fading rate of $\sim 2.2 \mathrm{mV} /$ cycle during 90 cycles compared to the LCD sample, $\sim 4.1 \mathrm{mV} /$ cycle (Figure S11). These comparisons indicate that different activation processes during the 1 st cycle strongly affect the electrochemical characteristics in subsequent cycles. Even though the two samples had similar amounts of cation disordering after the 1st cycle, the CD sample showed significantly improved electrochemical characteristics in subsequent cycles because it undergoes negligible structural changes and retains stable $\mathrm{Li}$ local environments during the $1^{\text {st }}$ cycle, whereas the LCD sample showed limited electrochemical characteristics because it undergoes severe structural changes during the activation process, and these changes can increase the electrochemically created cation disordering.

The different activation processes in the 1st cycle in 3d-TMbased Li-rich layered materials also substantially affect the electrochemical kinetics in subsequent cycles because of different degrees of structural changes after the activation process in the 1 st cycle. To understand the electrochemical kinetics, the galvanostatic intermittent titration technique (GITT) was performed for the samples after the 1st cycle (Figures $3 \mathrm{f}$ and S12). When the GITT technique is used, the cell is charged with a constant current of $5.6 \mathrm{~mA} / \mathrm{g}$ (C/50 rate) for $1 \mathrm{~h}$ and then relaxed for $2 \mathrm{~h}$ to reach a near-equilibrium state; this process is then repeated until the final cut-off voltage is reached. During the 1st charge, both samples showed increased polarization in the voltage range $>4.5 \mathrm{~V}$ or $>125$ $\mathrm{mAh} / \mathrm{g}$, where the oxygen redox reaction is mainly attributed (Figure 3f).4,11,37 However, the polarization above $4.5 \mathrm{~V}$ during the 1st charge is much lower in the CD sample than in the LCD sample (upper, Figure $3 \mathrm{f}$ ); this difference indicates that a facile oxygen redox reaction in the CD sample occurs even after almost full extraction of Li. Similarly, the polarization $<3.5 \mathrm{~V}$ or $>125 \mathrm{mAh} / \mathrm{g}$ during the 1 st discharge process, in which a charge compensation mainly occurs in the oxygen redox reaction, $4,11,37$ is much lower in the CD sample than in the LCD sample (lower, Figure 3f). This difference indicates that the oxygen redox reaction is kinetically easier in the CD sample than in the LCD sample. Furthermore, the changes in the diffusion coefficient $\mathrm{DLi}_{\mathrm{Li}}$ of $\mathrm{Li}$ ions for both samples during the 1st cycle (Figure S12) showed a similar tendency to the change in polarization (Figure 3f).

Especially, $D_{\text {Li above }} \sim 125 \mathrm{mAh} / \mathrm{g}$ during the 1 st discharge process is higher in the CD sample than in the LCD sample (Figure S12c). These results indicate that the kinetic properties of the oxygen redox reaction are significantly improved in the CD sample compared to the LCD sample. The oxygen redox reaction can be coupled to local structural changes, especially migration of TM ions; 9,10 therefore, the improved kinetics in the CD sample can originate from the negligible structural changes caused by the cation disordering in the pristine sample during the activation process in the $1^{\text {st }}$ cycle (Figure 2). Furthermore, the CD sample (Figure 3g) shows substantially improved rate capability in cycles after the activation process in the 1st cycle compared to the LCD sample, even though both samples have a similar amount of it after the 1st cycle; the cation disordering for the CD sample is from the pristine but that for the LCD sample is from the electrochemical reaction at the 1st cycle. A rate of $n \cdot C$ corresponds to a full discharge in $1 / n \mathrm{~h}$. The samples were charged at the $\mathrm{C} / 20$ rate and then discharged at various rates. The CD sample achieved $\sim 250 \mathrm{~mA} \cdot \mathrm{h} / \mathrm{g}$ of capacity at the $1 \mathrm{C}$ discharge rate $(280$ 
$\mathrm{mA} / \mathrm{g}$ ) and $\sim 210 \mathrm{~mA} \cdot \mathrm{h} / \mathrm{g}$ at the $3 \mathrm{C}$ discharge rate, whereas the LCD sample showed only $\sim 170 \mathrm{~mA} \cdot \mathrm{h} / \mathrm{g}$ at the $1 \mathrm{C}$ discharge rate and $\sim 120 \mathrm{~mA} \cdot \mathrm{h} / \mathrm{g}$ at the $3 \mathrm{C}$ discharge rate. Both samples received a similar contribution of $\sim 125 \mathrm{mAh} / \mathrm{g}$ from the Ni redox reaction to the obtained discharge capacity, but the CD sample showed much higher capacity even at the $3 \mathrm{C}$ rate than $\sim 125 \mathrm{mAh} / \mathrm{g}$, whereas the LCD sample did not. This difference indicates that the kinetics of the oxygen redox reaction, even at high rates in the CD sample, is significantly enhanced and can be comparable to the kinetics of the TM redox reaction. Even though the two samples have similar amounts of cation disordering after the 1st cycle, the different rate capability of the two samples in subsequent cycles suggests that their electrochemical kinetics depend strongly on the structural changes in the 1st cycle rather than the amount of it. Severe structural changes in the bulk and the surface caused by the activation process of the oxygen redox reaction during the 1st charge, like the LCD sample, easily led to poor kinetics. In contrast, negligible structural changes in the bulk and the surface enabled by the cation disordering in the pristine material like the CD sample can improve kinetics after the 1st cycle.

The above results clearly demonstrate that the structural changes during the activation process in the 1 st cycle strongly affect both the electrochemical activity in the subsequent cycles and the rate capability. Co-free 3d-TMbased Li-rich layered materials that undergo negligible structural changes during the 1st cycle can achieve the high electrochemical activity, sustainability, and remarkably improved rate capability, whereas the materials that undergo severe structural changes in the 1 st cycle do not achieve these improved electrochemical characteristics.

The cation disordering in the pristine sample in the 3d-TM-based Li-rich layered materials can be one of the factors for improving the activation process during the 1st cycle by enabling the tolerance to the structural changes in the activation process and, thereby, the resulting material can achieve high electrochemical activity and sustainability with remarkably improved kinetics in subsequent cycles.

Further Increase in the Structure Tolerance to the Structural Change Enabled by the Increase in the Pristine Cation Disordering: HCD Sample. We found out that the mitigation of structural changes during the 1 st cycle is a critical step to improve the electrochemical properties and kinetics of 3d-TM-based Li-rich layered materials in subsequent cycles, and this process can be improved by the cation disordering in the pristine materials. To achieve a further increase in the electrochemical activity and kinetics, the cation disordering in the pristine sample can be increased. We prepared the HCD (relatively high cation disordered) sample, which had the same nominal composition as the CD and LCD sample, but a higher degree of cation disordering in the pristine state than the $\mathrm{CD}$ sample. The quenching process was the same as used for the CD sample, but to increase the cation disordering in the pristine structure, a heating temperature was increased from 800 to $900{ }^{\circ} \mathrm{C}$ because the increase in the temperature increases the thermal energy, which determines the entropy contribution and the degree of the cation diffusion.

Rietveld refinement of NPD (Figure 4a, Table S1c) showed that the HCD sample is also composed of both R and M phases, like the LCD and CD samples. The refinement result (Figure 4b) showed that the HCD sample has a similar degree of cation disordering in the pristine ( $\mathrm{Ni}$ in the Li layer) in the $\mathrm{R}$ phase as the LCD and CD samples, but a much higher degree of cation disordering in the pristine sample in the $\mathrm{M}$ phase than the $\mathrm{CD}$ sample did. The amount of $\mathrm{Ni}$ in the Li layer of the R phase in the HCD sample is slightly increased: $\sim 0.074 \mathrm{~mol}$ for the HCD sample (Figure 4b, square) and $\sim 0.060 \mathrm{~mol}$ in the CD sample (Table S1b). However, the amount of $\mathrm{Ni}$ in the Li layer of the M phase in the HCD sample was substantially increased to $\sim 0.121$ mol (Figure $4 \mathrm{~b}$, star) compared to $\sim 0.080 \mathrm{~mol}$ in the $\mathrm{CD}$ sample (Table S1c). This comparison indicates that the cation disordering in the pristine sample was increased in the HCD sample compared to the CD sample and that the degree of cation disordering in the HCD pristine sample was highest among the three samples. The HCD sample also had a similar particle size $>200 \mathrm{~nm}$ and morphology as the CD and LCD samples (Figures 1b, 2b, and 4c). These results indicate that the HCD sample has a much higher degree of cation disordering in the pristine sample, especially in the M phase, than the LCD and CD samples, although all three samples had the same nominal composition and similar overall composition (Table S4). A further increase in the cation disordering in the pristine sample of the HCD sample strongly affected the electrochemical properties during the 1st cycle; the HCD sample showed further lowering of charge redox reaction potential and a further decrease in the voltage plateau at $\sim 4.5 \mathrm{~V}$ in the $1^{\text {st }}$ charge, compared to the $\mathrm{CD}$ sample, and higher discharge redox potential than the CD sample (red arrow in Figure 4d,e). These improved electrochemical properties indicate that the HCD sample undergoes less structural change than the CD sample during the 1st cycle. The HCD sample shows high 
reversible discharge capacities $(\sim 300 \mathrm{~mA} \cdot \mathrm{h} / \mathrm{g})$ with a high discharge voltage of $3.61 \mathrm{~V}$ even after almost full extraction of Li during the 1st charge; therefore, the energy density of the HCD sample in the 1st cycle is higher than in the CD sample. This difference indicates that a further increase in the cation disordering in the pristine sample can further improve electrochemical activity in the 1st cycle. The trend in the change of the redox voltage in the 1 st cycle was linearly related to the degree of the cation disordering in the pristine sample in the samples (red arrow in Figure 4d). This trend suggests that a further increase in the cation disordering in the pristine sample of 3d-TM-based Li-rich layered materials can strengthen the tolerance to the structural change that occurs during the activation process and yield further improvement in electrochemical activity.

The HCD sample also showed remarkably improved capacity retention (Figure 4f) and suppressed voltage fade (Figures $4 \mathrm{~g}$ and S11) during cycling compared to the LCD and CD samples (Figure 3e). The HCD sample showed a significantly improved capacity retention at the C/3 charge and C/3 discharge rate from $\sim 240$ to $\sim 200$ $\mathrm{mA} \cdot \mathrm{h} / \mathrm{g}(\sim 84 \%)$ after 100 cycles; the obtained capacities are much higher than the capacity from the Ni redox reaction $(\sim 125 \mathrm{mAh} / \mathrm{g})$, indicating that the sustainable oxygen redox reaction is significantly improved. The HCD sample achieves a very low voltage fading rate of $\sim 1.5 \mathrm{mV} /$ cycle during 100 cycles, compared to the LCD sample, which showed $\sim 4.1 \mathrm{mV} /$ cycle during 90 cycles (Figure $4 \mathrm{~g}$ ) and the CD sample, which showed $\sim 2.2$ $\mathrm{mV} /$ cycle during 90 cycles (Figure S11). This comparison indicates that structural coupling with the oxygen redox reaction during cycling can be substantially lowered in the HCD sample. 9

Given that the HCD sample had the highest degree of cation disordering in the pristine sample among the three samples, and the structural changes during the 1st cycle strongly depended on the pristine cation disordering that can suppress structural changes in the activation process, the improved capacity retention and suppressed voltage fade in the HCD material together confirmed that the increase in the cation disordering in the pristine sample substantially improved electrochemical activities such as achievable capacity and redox potentials in the 1 st cycle, and capacity retention and voltage fade upon cycling.
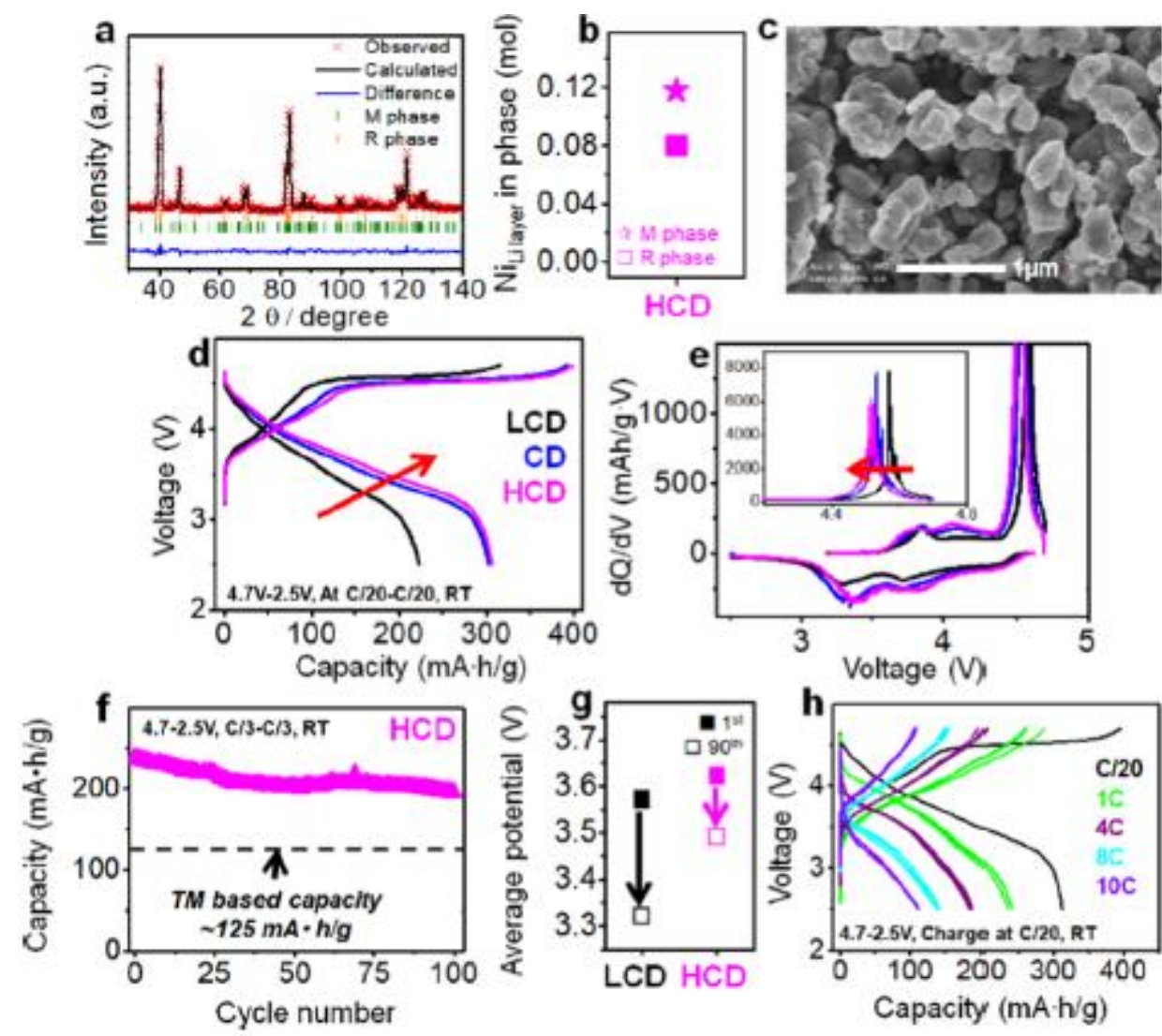

Figure 4. Structural/chemical characterization and electrochemical characteristics of the HCD sample. (a) Rietveld refinement of the neutron powder diffraction (NPD) result of the sample. Vertical whiskers: theoretical NPD patterns of (orange) the R phase (e.g., LiNio.5Mn0.5 $\mathrm{O}_{2}$ ) and (green) the $\mathrm{M}$ phase (e.g., $\mathrm{Li}_{2} \mathrm{MnO}_{3}$ ) (Table S1c). (b) Site occupancies of the Ni atoms in the 
Li layer of the $\mathrm{M}(\mathrm{C} / 2 \mathrm{~m})$ phase and the $\mathrm{R}(\mathrm{R}-3 \mathrm{~m})$ phase. (c) SEM image. The comparison of the 1st electrochemical properties of the HCD sample with others, (d) voltage profile and (e) $\mathrm{dQ} / \mathrm{dV}$ profile at $2.5-4.7 \mathrm{~V}$ and $\mathrm{C} / 20(14 \mathrm{~mA} / \mathrm{g})$ at $\mathrm{RT}$. (f) Capacity retention of the HCD sample at the $\mathrm{C} / 3$ rate for 100 cycles. (g) Changes in the average discharge potential (i.e., potential obtained at half-discharge capacity) at the $\mathrm{C} / 3$ rate for cycles. (h) Rate capability of the HCD sample at different discharge rates with the same charge rate at $\mathrm{C} / 20$. All electrochemical tests were performed at room temperature in an operating voltage window $2.5-4.7 \mathrm{~V}$.

The HCD sample also showed a higher rate capability (Figures 4h and S13) than the LCD and CD samples (Figure 3g) with respect to both achievable capacity and redox potential, even though the HCD sample has a higher degree of cation disordering in the pristine sample than the LCD and CD samples. The HCD sample achieved $\sim 250 \mathrm{~mA} \cdot \mathrm{h} / \mathrm{g}$ capacity at the $1 \mathrm{C}$ discharge rate $(280 \mathrm{~mA} / \mathrm{g}), \sim 200 \mathrm{~mA} \cdot \mathrm{h} / \mathrm{g}$ at the $4 \mathrm{C}$ discharge rate, and a reasonable capacity of $\sim 120 \mathrm{~mA} \cdot \mathrm{h} / \mathrm{g}$ even at the $10 \mathrm{C}$ discharge rate $(2800 \mathrm{~mA} / \mathrm{g})$ with increased redox potentials. Cation disordering has been believed to induce slow Li diffusion in the layered structure; $21,33,45,46$ therefore, the dramatically increased rate capability in the materials with the increase in cation disordering in the pristine sample is a counter-intuitive result. This unusual kinetic behavior can be understood by the less structural damage enabled by different activation processes during the 1st cycle, which is governed by the amount of cation disordering in the pristine sample in Li-rich materials. The cation disordering in the pristine in Co-free 3d-TM-based Lirich layered materials can enable the improved tolerance to the structural changes that occur during the 1st cycle; as a result, structural changes are negligible, and the Li local environments

change little after the 1st cycle. Even though the Li-rich materials have a certain degree of the cation disordering in the pristine sample before an electrochemical cycle, after the activation process in the 1st cycle, the 3d-TM based Li-rich layered materials with the cation disordering in the pristine sample can have less structural changes with enhanced tolerance to the structural changes. As a result, the 3d-TMbased Li-rich layered materials can achieve increased electrochemical activity and sustainability and superior rate capability in subsequent cycles. This analysis suggests that the way to activate the Li-rich layered materials in the 1st cycle is a critical factor that affects rate capability and electrochemical properties in the Li-rich layered materials and that introducing the cation disordering into the pristine sample can be one of the effective ways to improve the activation process in the 1st cycle via the improved tolerance to the structural changes.

\section{CONCLUSIONS}

In summary, we demonstrated that the activation process during the 1st cycle in $\mathrm{Li}_{2} \mathrm{MnO}_{3}$-based 3d-TM-based Li-rich layered materials has critical effects on their electrochemical characteristics such as electrochemical activity, sustainability, and rate capability upon cycling because it can govern the structural damages of materials after the 1st cycle. As a result, the activation process should be controlled for achieving high electrochemical performance. Also, the results suggest that the degree of the cation disordering in the pristine sample can be one of the effective ways to control the structural changes that occur during the activation process in the 1st cycle because the increased cation disordering in the pristine sample can improve tolerance to the structural changes that occur during the $1^{\text {st }}$ cycle, and thereby lead to negligible structural changes during the 1 st and subsequent cycles. As a result, 3d-TM-based Li-rich layered materials that had increased cation disordering in the pristine sample can achieve both high electrochemical activity in the 1st cycle and sustainable electrochemical characteristics in subsequent cycles. The findings and understandings will be exploited to effectively design $3 \mathrm{~d}$ TM-based Li-rich layered materials that achieve high sustainable energy density and superior rate capability.

\section{Supporting Information}

Rietveld refinement results, ex situ soft-XAS, ex situ Li NMR, STEM images, GITT, electrochemical performance; neutron powder diffraction (NPD) of LCD and CD samples; electrochemical properties of the LCD sample and ex situ XANES measurements for the electrodes prepared at different electrochemical cycles; ex situ soft-XAS measurements for the LCD electrodes prepared at different electrochemical cycles; structural changes of the LCD sample with STEM-ABF before/ after the 1st charge (PDF) 


\section{AUTHOR INFORMATION}

\section{Corresponding Author}

Byoungwoo Kang - Department of Materials Science and Engineering, Pohang University of Science and Technology (POSTECH), Pohang 37673, Republic of Korea;

orcid.org/0000-0002-8081-1908; Email: bwkang@ postech.ac.kr

Authors

Junghwa Lee - Department of Materials Science and Engineering, Pohang University of Science and Technology (POSTECH), Pohang 37673, Republic of Korea; Department of Materials Science and Engineering, Stanford University, Stanford, California 94305, United States

Yujeong Yang - Department of Materials Science and Engineering, Pohang University of Science and Technology (POSTECH), Pohang 37673, Republic of Korea

Mihee Jeong - Department of Energy Science, Sungkyunkwan University, Suwon 16419, Republic of

Korea; orcid.org/0000-0003-1700-2419

Nicolas Dupre - Université de Nantes, Institut des Materiaux Jean Rouxel (IMN), CNRS UMR 6502,

44322 Nantes Cedex 3, France; orcid.org/0000-0002-0687-9357

Maxim Avdeev - Australian Nuclear Science and Technology Organisation, Kirrawee DC, NSW 2232,

Australia; School of Chemistry, The University of Sydney, Sydney, NSW 2006, Australia;

orcid.org/0000-0003-2366-5809

Won-Sub Yoon - Department of Energy Science, Sungkyunkwan University, Suwon 16419, Republic of Korea; orcid.org/0000-0002-6922-2088

Si-Young Choi - Department of Materials Science and Engineering, Pohang University of Science and

Technology (POSTECH), Pohang 37673, Republic of Korea; orcid.org/0000-0003-1648-142X

\section{Author Contributions}

B.K. and J.L. designed all experiments. J.L. synthesized the materials and conducted all electrochemical measurements. Y.Y. and S.-Y.C. conducted all TEM characterization. M.J. and W.-S.Y. conducted hard X-ray XAS measurements. N.D. conducted hard Li NMR measurements. N.D. conducted Neutron measurements. All authors contributed to writing this manuscript.

\section{ACKNOWLEDGMENTS}

This research was supported by the Brain Korea 21 PLUS Project for Center for Creative Industrial Materials (F14SN02D1707) and a POSTECH Basic Science Research Institute Grant. This research was supported by the Basic Science Research Program through the National Research Foundation of Korea (NRF), funded by the Ministry of S c i e n c e, I C T \& F u t u r e P 1 a n n i n g (NR F - 2018M2A2B3A05074603 and NRF 2019R1A2C2007933).

\section{REFERENCES}

(1) Chu, S.; Cui, Y.; Liu, N. The path towards sustainable energy. Nat. Mater. 2017, 16, 16.

(2) Goodenough, J. B.; Kim, Y. Challenges for rechargeable Li batteries. Chem. Mater. 2010, 22, 587-603.

(3) Seo, D.-H.; Lee, J.; Urban, A.; Malik, R.; Kang, S.; Ceder, G. The structural and chemical origin of the oxygen redox activity in layered and cation-disordered Li-excess cathode materials. Nat. Chem. 2016, 8, 692.

(4) Luo, K.; Roberts, M. R.; Hao, R.; Guerrini, N.; Pickup, D. M.; Liu, Y.-S.; Edström, K.; Guo, J.; Chadwick, A. V.; Duda, L. C.; Bruce, P. G. Charge-compensation in 3d-transition-metal-oxide intercalation cathodes through the generation of localized electron holes on oxygen. Nat. Chem. 2016, 8, 684-691.

(5) Yabuuchi, N.; Nakayama, M.; Takeuchi, M.; Komaba, S.; Hashimoto, Y.; Mukai, T.; Shiiba, H.; Sato, K.; Kobayashi, Y.; Nakao, A.; et al. Origin of stabilization and destabilization in solid-state redox reaction of oxide ions for lithium-ion batteries. Nat. Commun. 2016, 7, No. 13814

(6) Oishi, M.; Yogi, C.; Watanabe, I.; Ohta, T.; Orikasa, Y.; Uchimoto, Y.; Ogumi, Z. Direct observation of reversible charge compensation by oxygen ion in $\mathrm{Li}$-rich manganese layered oxide positive electrode material, $\mathrm{Li} 1.16 \mathrm{Ni} 0.15 \mathrm{Co} 0.19 \mathrm{Mn} 0.50 \mathrm{O}_{2}$. J. Power Sources 2015, 276, 89-94.

(7) Mohanty, D.; Li, J.; Abraham, D. P.; Huq, A.; Payzant, E. A.; Wood, D. L., III; Daniel, C. Unraveling the voltage-fade mechanism in high-energy-density lithium-ion batteries: origin of the tetrahedral cations for spinel conversion. Chem.

Mater. 2014, 26, 6272-6280.

(8) Bettge, M.; Li, Y.; Gallagher, K.; Zhu, Y.; Wu, Q.; Lu, W.; Bloom, I.; Abraham, D. P. Voltage fade of layered oxides: its measurement and impact on energy density. J. Electrochem. Soc. 2013, 160, A2046-A2055. 
(9) Gent, W. E.; Lim, K.; Liang, Y.; Li, Q.; Barnes, T.; Ahn, S.-J.; Stone, K. H.; McIntire, M.; Hong, J.; Song, J. H.; et al. Coupling between oxygen redox and cation migration explains unusual electrochemistry in lithium-rich layered oxides. Nat. Commun. 2017, 8, No. 2091.

(10) Myeong, S.; Cho, W.; Jin, W.; Hwang, J.; Yoon, M.; Yoo, Y.; Nam, G.; Jang, H.; Han, J.-G.; Choi, N.-S.; et al. Understanding voltage decay in lithium-excess layered cathode materials through oxygen-centred structural arrangement. Nat. Commun. 2018, 9, No. 3285.

(11) Assat, G.; Foix, D.; Delacourt, C.; Iadecola, A.; Dedryvère, R.; Tarascon, J.-M. Fundamental interplay between anionic/cationic redox governing the kinetics and thermodynamics of lithium-rich cathodes. Nat. Commun. 2017, 8, No. 2219.

(12) Ates, M. N.; Mukerjee, S.; Abraham, K. A high rate Li-rich layered MNC cathode material for lithium-ion batteries. RSC Adv. 2015, 5, 27375-27386.

(13) Zheng, J.; Gu, M.; Xiao, J.; Polzin, B. J.; Yan, P.; Chen, X.; Wang, C.; Zhang, J.-G. Functioning mechanism of $\mathrm{AlF}_{3}$ coating on the Li-and Mn-rich cathode materials. Chem. Mater. 2014, 26, 6320-6327.

(14) Qiu, B.; Zhang, M.; Wu, L.; Wang, J.; Xia, Y.; Qian, D.; Liu, H.; Hy, S.; Chen, Y.; An, K.; et al. Gas-solid interfacial modification of oxygen activity in layered oxide cathodes for lithium-ion batteries. Nat. Commun. 2016, 7, No. 12108.

(15) Qiao, Q.-Q.; Qin, L.; Li, G.-R.; Wang, Y.-L.; Gao, X.-P. Snstabilized Li-rich layered Li(Lio.17Nio.25Mn0.58)O2 oxide as a cathode for advanced lithium-ion batteries. J. Mater. Chem. A 2015, 3, 17627- 17634.

(16) Sathiya, M.; Rousse, G.; Ramesha, K.; Laisa, C.; Vezin, H.; Sougrati, M. T.; Doublet, M.-L.; Foix, D.; Gonbeau, D.; Walker, W.; et al. Reversible anionic redox chemistry in high-capacity layeredoxide electrodes. Nat. Mater. 2013, 12, 827 835.

(17) Saubanère, M.; McCalla, E.; Tarascon, J.-M.; Doublet, M.-L. The intriguing question of anionic redox in high-energy density cathodes for Li-ion batteries. Energy Environ. Sci. 2016, 9, 984-991.

(18) Assat, G.; Tarascon, J.-M. Fundamental understanding and practical challenges of anionic redox activity in Li-ion batteries. Nat. Energy 2018, 3, 373-386.

(19) Lee, J.; Kitchaev, D. A.; Kwon, D.-H.; Lee, C.-W.; Papp, J. K.; Liu, Y.-S.; Lun, Z.; Clément, R. J.; Shi, T.; McCloskey, B. D.; et al. Reversible Mn 2+/Mn 4+ double redox in lithium-excess cathode materials. Nature 2018, 556, 185.

(20) Yabuuchi, N.; Takeuchi, M.; Nakayama, M.; Shiiba, H.; Ogawa, M.; Nakayama, K.; Ohta, T.; Endo, D.; Ozaki, T.; Inamasu, T.; et al. High-capacity electrode materials for rechargeable lithium batteries: Li3NbO4-based system with cationdisordered rocksalt structure. Proc. Natl. Acad. Sci. U.S.A. 2015, 112, 7650-7655.

(21) Lee, J.; Urban, A.; Li, X.; Su, D.; Hautier, G.; Ceder, G. Unlocking the potential of cation-disordered oxides for rechargeable lithium batteries. Science 2014, 343, 519-522.

(22) Myeong, S.; Cho, W.; Jin, W.; Hwang, J.; Yoon, M.; Yoo, Y.; Nam, G.; Jang, H.; Han, J.-G.; Choi, N.-S.; et al. Understanding voltage decay in lithium-excess layered cathode materials through oxygen-centred structural arrangement. Nat. Commun. 2018, 9, No. 587.

(23) Liu, S.; Liu, Z.; Shen, X.; Wang, X.; Liao, S. C.; Yu, R.; Wang, Z.; Hu, Z.; Chen, C. T.; Yu, X.; et al. Li-Ti Cation Mixing Enhanced Structural and Performance Stability of Li-Rich Layered Oxide. Adv. Energy Mater. 2019, 9, No. 1901530.

(24) Wu, Y.; Xie, L.; He, X.; Zhuo, L.; Wang, L.; Ming, J. Electrochemical activation, voltage decay and hysteresis of Lirich layered cathode probed by various cobalt content. Electrochim. Acta 2018, 265, 115-120.

(25) Laisa, C.; Kumar, A. N.; Chandrasekaran, S. S.; Murugan, P.; Lakshminarasimhan, N.; Govindaraj, R.; Ramesha, K. A comparative study on electrochemical cycling stability of lithium rich layered cathode materials $\mathrm{Li} 1.2 \mathrm{Ni} 0.13 \mathrm{M} 0.13 \mathrm{Mn}_{0.54} \mathrm{O}_{2}$ where $\mathrm{M}=\mathrm{Fe}$ or Co. J. Power Sources 2016, 324, 462-474.

(26) Zuo, Y.; Li, B.; Jiang, N.; Chu, W.; Zhang, H.; Zou, R.; Xia, D. A High-Capacity O2-Type Li-Rich Cathode Material with a Single- Layer $\mathrm{Li}_{2} \mathrm{MnO}_{3}$ Superstructure. Adv. Mater. 2018, 30, No. 1707255.

(27) Boulineau, A.; Simonin, L.; Colin, J.-F.; Bourbon, C.; Patoux, S. First evidence of manganese-nickel segregation and densification upon cycling in Li-rich layered oxides for lithium batteries. Nano Lett. 2013, 13, 3857-3863.

(28) Fell, C. R.; Qian, D.; Carroll, K. J.; Chi, M.; Jones, J. L.; Meng, Y. S. Correlation between oxygen vacancy, microstrain, and cation distribution in lithium-excess layered oxides during the first electrochemical cycle. Chem. Mater. 2013, 25, 162 -1629 .

(29) Lee, J.; Papp, J. K.; Clément, R. J.; Sallis, S.; Kwon, D.-H.; Shi, T.; Yang, W.; McCloskey, B. D.; Ceder, G. Mitigating oxygen loss to improve the cycling performance of high capacity cation-disordered cathode materials. Nat. Commun. 2017, 8, No. 981.

(30) Yan, P.; Nie, A.; Zheng, J.; Zhou, Y.; Lu, D.; Zhang, X.; Xu, R.; Belharouak, I.; Zu, X.; Xiao, J.; et al. Evolution of lattice structure and chemical composition of the surface reconstruction layer in $\mathrm{Li} 1.2 \mathrm{Ni} 0.2 \mathrm{Mn} 0.6 \mathrm{O}_{2}$ cathode material for lithium ion batteries. Nano Lett. 2015, 15, 514-522.

(31) Genevois, C.; Koga, H.; Croguennec, L.; Ménétrier, M.; Delmas, C.; Weill, F. Insight into the atomic structure of cycled lithium-rich layered oxide $\mathrm{Li}_{1} .20 \mathrm{Mn} 0.52 \mathrm{Co} 0.13 \mathrm{Ni}_{1}{ }_{13} \mathrm{O}_{2}$ using HAADF STEM and electron nanodiffraction. J. Phys. Chem. C 2015, 119, 75-83.

(32) Kim, S.; Cho, W.; Zhang, X.; Oshima, Y.; Choi, J. W. A stable lithium-rich surface structure for lithium-rich layered cathode materials. Nat. Commun. 2016, 7, No. 13598.

(33) Julien, C.; Mauger, A.; Zaghib, K.; Groult, H. Optimization of layered cathode materials for lithium-ion batteries. Materials 2016, 9, No. 595.

(34) Koga, H.; Croguennec, L.; Ménétrier, M.; Mannessiez, P.; Weill, F.; Delmas, C.; Belin, S. Operando X-ray absorption study of the redox processes involved upon cycling of the $\mathrm{Li}$-rich layered oxide $\mathrm{Li} 1.2 \mathrm{Mn} 0.54 \mathrm{Co} .13 \mathrm{Ni} 0.13 \mathrm{O}_{2}$ in $\mathrm{Li}$ ion batteries. J. Phys. Chem. C 2014, 118, 5700-5709. 
(35) Yoon, W.-S.; Balasubramanian, M.; Chung, K. Y.; Yang, X.-Q.; McBreen, J.; Grey, C. P.; Fischer, D. A. Investigation of the Charge Compensation Mechanism on the Electrochemically Li-Ion Deintercalated Li1-xCo1/3Ni1/3Mn $1 / 3 \mathrm{O}_{2}$ Electrode System by Combination of Soft and Hard X-ray Absorption Spectroscopy. J. Am. Chem. Soc. 2005, 127, $17479-17487$. (36) Yu, X.; Lyu, Y.; Gu, L.; Wu, H.; Bak, S. M.; Zhou, Y.; Amine, K.; Ehrlich, S. N.; Li, H.; Nam, K. W.; et al. Understanding the Rate Capabili ty of High-Energy-Densi ty Li-Rich Layered Li1.2Nio.15Co0.1Mn0.55 $\mathrm{O}_{2}$ Cathode Materials. Adv. Energy Mater. 2014, 4, No. 1300950.

(37) Kaewmala, S.; Limphirat, W.; Yordsri, V.; Kim, H.; Muhammad, S.; Yoon, W.-S.; Srilomsak, S.; Limthongkul, P.; Meethong, N. Structural and Electrochemical Kinetic Properties of $0.5 \mathrm{Li}_{2} \mathrm{MnO}_{3} \cdot 0.5 \mathrm{LiCoO}_{2}$ Cathode Materials with Different Li2 $\mathrm{MnO}_{3}$ Domain Sizes. Sci. Rep. 2019, 9, No. 107326.

(38) Jin, W.; Myeong, S.; Hwang, J.; Jang, H.; Sung, J.; Yoo, Y.; Kim, M. G.; Cho, J. Unraveling the Rapid Redox Behavior of Li-Excess 3d- Transition Metal Oxides for High Rate Capability. Adv. Energy Mater.

2020, 10, No. 1904092.

(39) Chung, S.-Y.; Choi, S.-Y.; Lee, S.; Ikuhara, Y. Distinct configurations of antisite defects in ordered metal phosphates: comparison between LiMnPO4 and LiFePO4. Phys. Rev. Lett. 2012, 108, No. 195501.

(40) Dogan, F.; Long, B. R.; Croy, J. R.; Gallagher, K. G.; Iddir, H.; Russell, J. T.; Balasubramanian, M.; Key, B. Re-entrant lithium local environments and defect driven electrochemistry of Li-and Mn-rich Li-ion battery cathodes. J. Am. Chem. Soc. $2015,137,2328-2335$.

(41) Buzlukov, A.; Mouesca, J.-M.; Buannic, L.; Hediger, S.; Simonin, L.; Canevet, E.; Colin, J.-F.; Gutel, T.; Bardet, M. LiRich Mn/Ni Layered Oxide as Electrode Material for Lithium Batteries: A 7Li MAS NMR Study Revealing Segregation into (Nanoscale) Domains with Highly Different Electrochemical Behaviors. J. Phys. Chem. C 2016, 120, 19049-19063.

(42) Singer, A.; Zhang, M.; Hy, S.; Cela, D.; Fang, C.; Wynn, T.; Qiu, B.; Xia, Y.; Liu, Z.; Ulvestad, A.; et al. Nucleation of dislocations and their dynamics in layered oxide cathode materials during battery charging. Nat. Energy 2018, 3, 641-647. (43) Dai, K.; Wu, J.; Zhuo, Z.; Li, Q.; Sallis, S.; Mao, J.; Ai, G.; Sun, C.; Li, Z.; Gent, W. E.; et al. High reversibility of lattice oxygen redox quantified by direct bulk probes of both anionic and cationic redox reactions. Joule 2019, 3, 518-541. (44) Muhammad, S.; Kim, H.; Kim, Y.; Kim, D.; Song, J. H.; Yoon, J.; Park, J.-H.; Ahn, S.-J.; Kang, S.-H.; Thackeray, M. M.; et al. Evidence of reversible oxygen participation in anomalously high capacity Li-and Mn-rich cathodes for Li-ion batteries. Nano Energy 2016, 21, 172-184.

(45) Huang, J.; Liu, H.; Hu, T.; Meng, Y. S.; Luo, J. Enhancing the electrochemical performance of Li-rich layered oxide $\mathrm{Li}_{1.13} \mathrm{Ni} 0.3 \mathrm{Mn} 0.57 \mathrm{O}_{2}$ via $\mathrm{WO}_{3}$ doping and accompanying spontaneous surface phase formation. J. Power Sources 2018, 375 , 21-28.

(46) Julien, C. M.; Mauger, A.; Zaghib, K.; Groult, H. Comparative issues of cathode materials for Li-ion batteries. Inorganics 2014, 2, 132-154. 